\title{
Audio-vestibular phenotypes and advanced magnetic resonance imaging features of Cochlin gene mutations carriers
}

Giorgio Conte M.D. ${ }^{1}$, Francesco Lo Russo M.D. ${ }^{2 *}$, Luca Caschera M.D. ${ }^{2}$, Diego Zanetti M.D. ${ }^{3}$, Pierangela Castorina M.D. ${ }^{3}$, Clara Sina M.D. ${ }^{1}$, Fabio Triulzi M.D. ${ }^{1,4}$, Federica Di Berardino M.D. ${ }^{3}$

${ }^{1}$ Fondazione IRCCS Ca' Granda Ospedale Maggiore Policlinico, Department of Neuroradiology, Milan, Italy.

${ }^{2}$ Università degli Studi di Milano, Postgraduation School of Radiodiagnostics, Milan, Italy.

${ }^{3}$ Fondazione IRCCS Ca' Granda Ospedale Maggiore Policlinico, Audiology Unit, Department of Clinical Sciences and Community, Università degli Studi di Milano, Milan, Italy.

${ }^{4}$ Università degli Studi di Milano, Department of Pathophysiology and Transplantation, Milan, Italy.

Short Title: Cochlin-related deafness

*Corresponding Author

Dr. Francesco Maria Lo Russo M.D.

Università degli Studi di Milano

Postgraduation School of Radiodiagnostics

Via Festa del Perdono 7

Milan, 20122, Italy

Tel: 00390255035160

E-mail: francesco.maria.lorusso@gmail.com

Keywords: $\mathrm{COCH}$ protein; deafness; magnetic resonance imaging; inner ear 


\section{Abstract}

Objective: to describe clinical and imaging findings in a group of patients affected by non-syndromic deafness A9 (DFNA9), using advanced Magnetic Resonance (MR) imaging with three-dimensional (3D) Fluid-Attenuated Inversion Recovery (FLAIR) sequence.

Method: a retrospective case review was conducted in a tertiary referral center in Italy. Four sequential adult DFNA9-affected patients, who had undergone MR imaging at our Department between January 2017 and June 2018, were enrolled (Male $=2$, Female $=2$; median age: 65,6yr; 8 diseased ears analyzed). Three patients were relatives; the fourth was unrelated. The main outcome measures - age, sex, records of audiological and vestibular testing, genetic assessment, MR imaging findings - were analyzed.

Results: All subjects suffered from bilateral progressive sensorineural hearing loss, more severe on the high frequencies and typical clinical pattern of bilateral chronic degenerative cochleo-vestibular deficit. Aural fullness was reported at the onset of the disease. All patient revealed a pathogenic heterozygous mutation in the Limulus factor $\mathrm{C}$, Coch- $5 \mathrm{~b} 2$ and Lgl1 domain (LCCL) of cochlin. None of the patients showed a significant vestibular and cochlear endolymphatic hydrops at MR imaging, while high bilateral contrast enhancement on 4hs-delayed postcontrast 3D-FLAIR sequence was observed in all ears.

Conclusions: increased perilymph enhancement on 4hs-delayed postcontrast 3D- FLAIR sequence is the common imaging feature of DFNA9 ears, suggesting that blood-labyrinthine barrier (BLB) breakdown may play the main role in the pathophysiology of this disease. Significant hydrops has been excluded by MR imaging. This finding might be clinically useful in differentiating DFNA9 disease from other pathologies with similar clinical findings like Ménière's disease. 


\section{Introduction}

Non-syndromic deafness A9 (DFNA9) is a cause of autosomal dominant late-onset sensorineural hearing loss (SNHL) associated with progressive bilateral vestibular deterioration [Manolis et al., 1996; Kemperman et al., 2005]. The disease starts with a high-frequency hearing loss and progresses to a poor hearing performance in the 6th and 7th decades [Manolis et al., 1996; Fransen, and Van Camp, 1999; Lemaire et al., 2003; Bischoff et al., 2005; Kemperman et al., 2005; Hildebrand et al., 2010]. Given the rarity of the disease [Fransen and Van Camp, 1999; Usami et al., 2003; Chen et al., 2013] and its clinical presentation characterized by fluctuating hearing loss accompanied with intermittent vertigo and dizziness, aural fullness and tinnitus, DFNA9 can sometimes be misdiagnosed as Ménière's disease or dehiscent superior semicircular canal syndrome [De Kok et al., 1999; Lemaire et al., 2003; Hildebrand et al., 2009]. Mutations in the $\mathrm{COCH}$ (Coagulation Factor C Homology) gene, found on the long arm of chromosome 14 (14q12-q13), has been linked to the development of the disease [Manolis et al., 1996]. The gene encodes for the cochlin protein, which is highly expressed in the cochlea and in the vestibular system [Robertson et al., 1998; Li et al., 2005; Robertson et al., 2006]. The function of cochlin has yet to be fully understood; it is believed to assist the local immunological response and to provide structural support in the inner ear [Bhattacharya, 2006; Py et al., 2013; Bae et al., 2014]. Although interest is increasing in the genomics and pathophysiology underlying $\mathrm{COCH}$-related disease and DFNA9, information about the morphological appearance at imaging are limited. Recent contrast-enhanced Magnetic Resonance (MR) imaging, with three-dimensional (3D) Fluid-Attenuated Inversion Recovery (FLAIR) sequence, allows in-vivo assessment of the Blood-Labyrinthine Barrier (BLB) permeability, of the content and relative volume of the perilymphatic compartment and of the morphology of the endolymphatic space (ES) in humans [Conte et al., 2017]. Our aim was to describe the imaging findings in a group of consecutive patients affected by DFNA9, using advanced MR imaging with 3D-FLAIR sequence.

\section{Materials and Methods}

\subsection{Subjects}

This study was approved by the Institutional Review Board of Fondazione IRCCS Ca' Granda Ospedale Maggiore Policlinico of Milan, Italy (Protocol N. 473_2018, accepted 19th June 2018). Informed consent was waived. We retrospectively enrolled all sequential patients affected by DFNA9, who had undergone MR imaging at our Department between January 2017 and June 2018. We also collected 24 consecutive MR imaging scans of normal ears (males $=13$, females $=11$; age range: $25-74$ years, median age: 53 years) obtained at our Department between July 2016 and December 2017, in order to constitute a control group. These "normal" ears were represented by the contralateral ear of a group of unilateral sudden deafness patients, diagnosed according to the American Academy of Otolaryngology - Head and Neck Surgery criteria [American Speech-Language-Hearing Association. Guidelines for Audiologic Screening [Guidelines], 1997], who had undergone MRI. From an otological viewpoint, ears were defined as normal according to the following criteria: 1) no history of otological disorders (either middle or inner ear pathology), no report of previous ear surgery and/or no history of previous or current vertigo episodes and/or dizziness, as evidenced by the patient's medical record; 2) normal results at the audiological evaluation with otomicroscopy, pure-tone audiometry, immittance audiometry, transient-otoacoustic emissions, and speech audiometry 3 ) no pathological findings on MRI.

\subsection{Audiological and vestibular assessment}

The measurements were all conducted in a sound-attenuated booth fulfilling the requirements of ISO 8253-3 (2012), at the Audiology Unit, Department of Surgical Sciences, University of Milan, Milan, Italy. 
After gathering all relevant clinical information about the onset and progression of hearing loss and any concurrent balance disorders, all subjects underwent the following battery of tests:

- otomicroscopy

- pure-tone audiometry (Amplaid A321, Resonance, Gazzaniga, Italy) with measurement of air conduction (AC) thresholds at all octave frequencies between 125 and $8000 \mathrm{~Hz}$ and of bone conduction (BC) thresholds between 250 and $4000 \mathrm{~Hz}$; thresholds were reported as PTA4 = pure tone average of $0.5-1-2-4 \mathrm{kHz}$

- middle ear impedance testing

- transiently evoked otoacoustic emissions (TEOAE) (Grason-Stadler Audera, Eden Prairie MN, USA)

- speech perception tests: disyllabic words recognition (WRS) delivered to each ear via TDH49 ear muffs (Amplaid A321, Resonance, Gazzaniga, Italy).

All patients with DFNA9 underwent also a vestibular assessment by means of:

- cervical and ocular vestibular evoked myogenic potentials (cVEMPS/oVEMPS, Amplaid MK22, Milano, Italy),

- static stabilometry (SVEP system 6.0, Amplaid, Politecnica Modena, Italy) at $5 \mathrm{~Hz}$ with eyes opened and closed, with and without foam pads (Di Berardino F., et al. The assessment of static posturography's variability and the sensorial ratios by the use of foam pads. Gait and Posture 2009: 29: 1;158-160).

- bithermal caloric testing, according to the Hallpike technique and Jongkee's formulae

- the recording of the vestibulo-ocular reflex (VOR) by video-oculography (VN 525, Interacoustics, Middelfart, Denmark) using a sinusoidal rotatory chair testing (burst of sinewave for $20 \mathrm{~s}$ ) with $30 \% \mathrm{sec}$ and $60 \% \mathrm{sec}$ peak chair velocity

- video head impulse test (v-HIT) (Otometrics Natus, Taastrup, Denmark). We used a gain cutoff value of 0.8 for the VHIT and presence of correction saccades to define an abnormal vestibular-ocular reflex on the horizontal semicircular canals.

\subsection{Genetic assessment}

DNA was extracted from peripheral blood samples using QIAamp DNA Mini Kit following the protocol provided by the manufacturer. Library preparation was performed with the TruSight One sequencing panel (Illumina, San Diego, USA), which enriches the sample for about 4,800 genes of clinical relevance (only exonic region). Massively parallel sequencing was conducted with NextSeq 5500 (Illumina, USA), Paired End method (2X151cycles). BaseSpace software (Illumina) allowed us to generate bam, bam.bai and vcf files and VariantStudio software ${ }^{\circledR}$ (Illumina(C) to analyze the files focused on 80 genes harboring known deafness-causing mutations including DFNA9. GRCh37 (hg19) was used as the reference sequence for mapping and variant calling. Databases used for analysis and variant annotation include the Online Mendelian Inheritance in Man (OMIM), Human Gene Mutation Database, ClinVar, Single Nucleotide Polymorphism database (dbSNP), Exome Aggregation Consortium (EXAC) and Exome Sequencing Project, with a minor allele frequency cut-off of $1 \%$. 


\subsection{MR imaging acquisition and assessment}

All patients were imaged on a 3 Tesla scanner (Achieva, Philips Healthcare, Netherlands) using a 32channel head coil. The imaging protocol consisted of sequences for both the whole brain and temporal bone assessment, as recommended in MR imaging of SSHL [Conte et al., 2018 a]. In addition, after intravenous contrast-agent administration, the protocol included 4 hours delayed post-contrast MR imaging for the evaluation of the membranous labyrinth as accepted in the recent literature [Nakashima et al., 2009; Conte et al., 2017; Conte et al., 2018 b; Conte et al., 2018 c]. Contrast-agent (Gadoteridol, Prohance ${ }^{\mathrm{TM}}$, Bracco Diagnostic Inc., Milan, Italy) was administered intravenously at the recommended dose $(0.2 \mathrm{mmol} / \mathrm{kg})$. MR protocol parameters are listed in Table 1. A neuroradiologist with 6 years of experience in otoradiology and a senior radiology resident, assessed MR images in consensus. For each ear the readers assessed: 1) on T2 weighted threedimensional (3D) steady-state free precession (FFSP) sequence the traverse section of the cochlear nerve on the para-sagittal plane perpendicular to the internal auditory canal at the level of the fundus, defining it as "normal" if larger than the facial nerve, "hypoplastic" if equal or less [Heining et al., 2017] (Figure 1a, b); 2) the cochlear signal on precontrast T1-weighted fast spin echo (T1 FSE), 3D T2-FFSP and 3D-FLAIR; pre-contrast T1 FSE and 3D-FLAIR sequences were performed to evaluate the eventual presence of blood product and/or proteinaceous content in the cochlea respectively; the enhancement on 4 hs-delayed postcontrast 3D-FLAIR sequence was measured as the ratio between the mean value of a polygonal region-of-interest (ROI) drawn on the middle turn the cochlea (ROI area $\cong 6 \mathrm{~mm} 3$ ) and the mean value of a circular ROI drawn on the cerebrospinal fluid (CSF) at the level of the cerebellar-pontine angle (Figure 2); these ratios were assessed for normal distribution with Shapiro-Wilk test and further compared with the distribution of the corresponding ratios calculated in the 24 healthy ears. Values were defined as not normal if felt outside \pm 2 standard deviations from the mean, in particular "decreased" if below 2 standard deviations from the mean (2.5 percentile), "increased" when above 2 standard deviations from the mean ( 97.5 percentiles), "normal" if comprised between 2 standard deviations from the mean (between the 2.5 and 97.5 percentiles); 3) the presence of vestibular (VEH) or cochlear endolymphatic hydrops (cEH) defined using the Nakashima's quantitative method on 4hs-delayed postcontrast 3D-FLAIR sequence [Nakashima et al., 2009] (Figure 2).

\section{Results}

Four DFNA9-affected patients were enrolled (male $=2$, female $=2$; median age: $65,6 \mathrm{yr}$ ) with a total of 8 diseased ears analyzed. Three patients were relatives; the fourth was unrelated and had no relatives to be tested. Control SSHL group counted 24 patients (males $=13$, females $=11$; median age: 53) with total of 24 healthy ears analyzed. All selected DFNA9 patients were white Caucasians, and for all of them, NGS revealed a pathogenic heterozygous mutation in the Limulus factor C, Coch$5 \mathrm{~b} 2$ and Lgl1 domain (LCCL) of cochlin. The genetic profile of DFNA9 patients is reported in Table 2; the missense heterozygous mutation in the $\mathrm{COCH}$ gene (NM_004086.2) was G88A in three relatives and $W 117 R$ in the other patient. The results of the audiological assessment are summarized in Table 3. All the DFNA9 subjects suffered from bilateral progressive SNHL, more severe on the high frequencies, tinnitus, and bilateral symmetrical labyrinthine hyporeflexia, embodying the typical clinical pattern of bilateral chronic degenerative cochleo-vestibular deficit. All patients complained of aural fullness from the onset of the disease, but none of them reported fluctuating hearing loss. The degree of hearing loss was moderately severe in two (one of them reported environmental exposure to acoustic trauma) and severe in two others. TEOAE were not detectable in all cases. Vestibular dysfunction was observed in all DFNA9 patients: it was characterized by weakened bilateral semicircular canal function (areflexia in one out of four patients) by both VOR, v-HIT and caloric testing. Despite the poor or very poor bilateral response at caloric testing, the two males (patient \#2 and 4) did not complain of vertigo spells and showed static posturography values within the normal range without foam pads, higher values showing static instability were found on foam pads especially 
at closed eyes. The acoustic reflexes were elicited with low thresholds (positive Metz test), supporting a probable cochlear origin of the hearing loss. Patients \#2, 3, 4 benefited from bilateral hearing aids $(\mathrm{HA})$ with customized earmolds. A more severe clinical pattern was observed in patient $\# 1$, who showed bilateral severe to profound SNHL with a longer duration of the disease and a higher frequency of vertigo spells and of episodes of sudden drops of hearing. She obtained limited benefit by $\mathrm{HA}$, thus underwent cochlear implantation $(\mathrm{Cl})$ in the right ear at our Department in December 2017. She retained her HA in the ear opposite to the $\mathrm{Cl}$, achieving a significant improvement of the perceptive abilities already after 6 months of bimodal hearing. The results of the imaging assessment are summarized in Table 4. Patient \#1 was the only one to show a high precontrast T1-signal intensity in the left ear. The same patient had a high precontrast FLAIR signal intensity in both ears. Another patient (\#3) showed high precontrast FLAIR signal intensity in the right ear and hypoplasia of the contralateral cochlear nerve. Patient \#4 showed hypoplasia of the left cochlear nerve but no other signal abnormalities on pre-contrast imaging. As in the control group, none of the DFNA9 patients showed a significant VEH and cEH. Conversely, all DFNA9 ears showed a high bilateral contrast enhancement on 4 hs-delayed postcontrast 3D-FLAIR sequence (Figure $2 \mathrm{~A}$ ).

\section{Discussion}

Our study showed that the common imaging finding in DFNA9 ears is the higher enhancement of the perilymph, in both cochlear and vestibular spaces, on 4hs-delayed post-contrast 3D FLAIR sequence. In addition, an increased signal on precontrast 3D-FLAIR was also observed in our patient \#1 and \#3; patient \#1 also showed increased signal on precontrast T1-weighted sequence. No signs of endolymphatic hydrops were identified in any of the 4 patients. At the best of our knowledge there are no studies that have already investigated MR findings of DFNA9 ears by a 3 Tesla scanner and using pre- and postcontrast 3D-FLAIR sequences, which has been demonstrated to be an emerging technique able to add new insights about different inner ear pathologies, such as sudden hearing loss and Ménière's disease [Conte et al., 2017]. Another study had attempted to assess the morphology of DFNA9 ears by computed tomography and by MRI using classic heavily-T2 MR sequence, which is, unfortunately, less sensitive in detecting the changes in inner ear structures [De Varebeke et al., 2014].

The increased enhancement of the perilymph on 4hs-delayed postcontrast 3D-FLAIR sequence suggests the breakdown of the blood-labyrinthine barrier (BLB). The BLB is crucial for the maintenance of inner ear fluid ionic homeostasis, preventing the entry of toxic substances into the inner ear. By studying pharmacokinetics of drugs and their distribution from the systemic circulation into the fluids of the inner ear, it has been demonstrated that BLB is highly selective and that the composition of the inner ear fluids is regulated and markedly different from that of blood or of other fluids such as cerebrospinal fluid [Juhn et al., 2001; Ishiyama et al., 2017]. It is well known that after its intravenous administration, the contrast-agent starts physiologically to accumulate in the perilymph, but not in the endolymph, crossing the BLB and reaching the maximum concentration at about 4.5 hours from the administration [Naganawa et al., 2012]. As a consequence, post-contrast MR imaging acquired at about 4-5 hours can distinguish the membranous labyrinth (hypointense) from the surrounding perilymph (hyperintense). In normal conditions the progressive enhancement of the perilymph should be symmetric, while an asymmetric enhancement at early and/or delayed post-contrast sequences indicates the BLB breakdown on the affected side [Berrettini et al., 2013; Pakdaman et al., 2016; Conte et al., 2017; Conte et al 2018]. In the three ears in which the enhancement is more evident (Table 4), the association of spontaneously increased signal on precontrast FLAIR suggests that the BLB breakdown caused the accumulation of proteinaceous material in the perilymph, among which, possibly also cochlin. Cochlin, expressed in the osseous spiral lamina, spiral ligament, limbus, and stroma of the maculae and cristae [Robertson et al., 1998; Li et al., 2005; Robertson et al., 2006], is a secreted protein with three functional domains (LCCL domain, vWFA1, VWFA2) likely to be involved in antibody-independent host defense mechanisms [Bhattacharya, 
2006; Py et al., 2013; Bae et al., 2014] and to be associated with extracellular matrix proteins, typically fibrillar collagens [Khetarpal, 2000; Nagy et al., 2008;]. Histopathologic studies revealed that in DFNA9 patients cochlin protein aggregates in the extracellular matrix, altering protein to protein interactions [Robertson et al., 1998; Merchant et al., 2000; Khetarpal, 2000; Street et al., 2005; Bhattacharya, 2006; Robertson et al., 2006; Bae et al., 2014], and possibly interfering with ionic homeostasis; it also accumulates in the spiral ligament, in the distal osseous spiral lamina, at the base of the spiral limbus and in mesenchymal tissue at the base of the vestibular neuroepithelium [Burgess et al., 2016]. An electron microscopic study by Khetarpal et al. [2000] demonstrated that mutated cochlin can alter normal interaction between fibrillar collagen type II and extracellular matrix in the spiral ligament, promoting collagen degradation [Khetarpal, 2000]. A direct toxic effect of the deposited material may cause severe degeneration of multiple cytologic elements [Merchant et al., 2000; Robertson et al., 2006; Burgess et al., 2016]. Moreover, the cochlin von Willebrand domains have been suggested to play a key role in increasing shear-induced platelet aggregation [Bhattacharya, 2006], possibly resulting in vascular disease with subsequent tissue degeneration. The direct toxic effect of protein aggregates and the induced vascular degeneration may play a causative role in the BLB breakdown. Interestingly, endolymphatic hydrops was not a prominent feature DFNA9 affected patient, despite vestibular symptoms (patient \#1 and 2), bilateral hyporeflexia (patient \#1, 3 and 4) or areflexia (patient \#2), and abnormal vestibular reflexes. This finding seems to suggest that the pathogenesis and the progression of otovestibular symptoms in DFNA9 are not determined by vestibular endolymphatic hydrops. Likewise, cochlear hydrops should not be addressed as the culprit behind progressive SNHL. DFNA9 has often been confused with Ménière's disease, due to similar clinical presentation especially in early stages [De Kok et al., 1999; Lemaire et al., 2003; Hildebrand et al., 2009]. In contrast, DFNA9 does not show signs of EH, which is instead a prominent pathophysiologic feature of Ménière's disease [Nakashima et al., 2009; Baráth et al., 2014]. As far as we know, this is the first study providing imaging evidence of absence of cEH and $\mathrm{VEH}$, in agreement and support to a previous report by Usami et al. [2003] who underlined the discrepancies between DFNA9 and Ménière's disease [Usami et al., 2003]. Our patients \#3 and \#4 showed unilateral hypoplasia of the cochlear nerve a finding which was not mentioned in previous DFNA9 studies. Hypoplasia of the cochlear nerve has been linked to profound deafness and reduced spiral ganglion cell count [Nadol and Xu, 1992; Kujawa and Liberman, 2009]. Interestingly, the reduced cochlear nerve diameter did not correlate with worse clinical performance (patient \#3 and 4). It should be kept in mind that the variability of diameters of cochlear nerves is wide in both hearing and deaf subjects [Nadol and $\mathrm{Xu}, 1992$ ]. Nevertheless, our patients achieved significant benefit from hearing aid amplification and cochlear implantation [Vermeire et al., 2006] despite DFNA9 is known to involve cochlear dendrites. Some limitations of our study should be acknowledged. Although the population being referred to our tertiary health care center is quite large, the number of identified and enrolled DFNA9 cases is small, reflecting the low-incidence of this pathology in the general population [Fransen and Van Camp, 1999; Usami et al., 2003; Chen et al., 2013]; nonetheless 3 out of 4 enrolled DFNA9 patients belonged to the same family and all the patients had long-standing disease with moderate-severe sensorineural hearing loss with vestibular hyporeflexia. Thus, our study could not rule out the possibility that clinical and MRI features of EH are present exclusively at the onset and in milder phenotypes. Future studies including larger cohorts with a broader clinical and genetic spectrum of DFNA9 are warranted to determine early and milder imaging phenotypes. We could not provide histological data that would have better explained our MR findings, which is another limitation that has to be cited.

\section{Conclusions}

Increased perilymph enhancement on 4hs-delayed postcontrast 3D-FLAIR sequence is the common imaging feature of DFNA9 ears, suggesting that BLB breakdown may play the main role in the pathophysiology of this disease. In contrast, MR imaging excludes the presence of significant hydrops. This finding might be clinically useful in differentiating DFNA9 disease mimicking Ménière's 
disease. Based on this report, further studies should evaluate if different DFNA9 mutations show similar MRI patterns with 4hs-delayed postcontrast 3D-FLAIR sequence. 


\section{Statements}

\subsection{Acknowledgement}

The authors are grateful to Eurofins Genoma Group S.r.L. Italy for the genetical laboratory analysis.

\subsection{Statement of Ethics}

This study was approved by the Institutional Review Board of Fondazione IRCCS Ca' Granda Ospedale Maggiore Policlinico of Milan, Italy (Protocol N. 473_2018, accepted 19th June 2018). Informed consent was waived for all subjects enrolled in the study.

\subsection{Disclosure Statement}

The authors have no conflicts of interest to declare.

\subsection{Funding Sources}

No funding has been received for the submitted work.

\subsection{Author Contributions}

G.C., F.D.B. and D.Z. conceived the study; F.M.L.R., L.C., F.D.B., and D.Z. analyzed the data; G.C., F.M.L.R., L.C., F.D.B., and D.Z contributed to the interpretation of the results; G.C. took the lead in writing the manuscript. All authors provided critical feedback and helped shape the research, analysis and manuscript. 


\section{References (Alphabetical)}

American Speech-Language-Hearing Association. Guidelines for Audiologic Screening [Guidelines] 1997; DOI: 10.1044/policy.GL1997-00199

Bae SH, Robertson NG, Cho HJ, Morton CC, Jung DJ, Baek JI, et al. Identification of pathogenic mechanisms of COCH mutations, abolished cochlin secretion, and intracellular aggregate formation: genotype-phenotype correlations in DFNA9 deafness and vestibular disorder. Hum Mutat 2014;35:1506-1513.

Baráth K, Schuknecht B, Monge Naldi A, Schrepfer T, Bockisch CJ, Hegemann SCA. Detection and grading of endolymphatic hydrops in Menière disease using MR imaging. Am J Neuroradiol 2014;35:1387-1392.

Berrettini S, Seccia V, Fortunato S, et al. Analysis of the 3-dimensional fluid-attenuated inversion-recovery (3D-FLAIR) sequence in idiopathic sudden sensorineural hearing loss. JAMA Otolaryngol Head Neck Surg. 2013 May;139(5):45664.

Bhattacharya SK. Focus on Molecules: Cochlin. Exp Eye Res 2006;82:355-356.

Bischoff AMLC, Huygen PLM, Kemperman MH, Pennings RJE, Bom SJH, Verhagen WIM, et al. Vestibular deterioration precedes hearing deterioration in the P51S COCH mutation (DFNA9): an analysis in 74 mutation carriers. Otol Neurotol 2005;26:918-925.

Burgess BJ, O'Malley JT, Kamakura T, Kristiansen K, Robertson NG, Morton CC, et al. Histopathology of the Human Inner Ear in the p.L114P COCH Mutation (DFNA9). Audiol Neurotol 2016;21:88-97.

Chen D-Y, Chai Y-C, Yang T, Wu H. Clinical characterization of a novel COCH mutation G87V in a Chinese DFNA9 family. Int J Pediatr Otorhinolaryngol 2013;77:1711-1715.

Conte G, Di Berardino F, Sina C, Zanetti D, Scola E, Gavagna C, et al. MR imaging in sudden sensorineural hearing loss. Time to talk. Am J Neuroradiol 2017;38:1475-1479.

Conte G., Caschera L., Calloni S, et al. MR Imaging in Menière Disease: Is the Contact between the Vestibular Endolymphatic Space and the Oval Window a Reliable Biomarker? Am J Neuroradiol 2018;39(11):2114-2119.

Conte G., Caschera L., Tuscano B, et al. Three-Tesla magnetic resonance imaging of the vestibular endolymphatic space: A systematic qualitative description in healthy ears. Eur J Radiol. 2018;109:77-82.

Conte G, Lo Russo FM, Calloni SF et al. MR imaging of endolymphatic hydrops in Ménière's disease: not all that glitters is gold. Acta Otorhinolaryngol Ital. 2018 Aug;38(4):369-376.

De Kok YJM, Bom SJH, Brunt TM, Kemperman MH, Van Beusekom E, Van Der Velde-Visser SD, et al. A Pro51Ser mutation in the $\mathrm{COCH}$ gene is associated with late onset autosomal dominant progressive sensorineural hearing loss with vestibular defects. Hum Mol Genet 1999;8:361-366.

De Varebeke SPJ, Termote B, Van Camp G, Govaerts PJ, Schepers S, Cox T, et al. Focal sclerosis of semicircular canals with severe dfna9 hearing impairment caused by a P51S COCH-mutation: Is there a link? Otol Neurotol 2014;35:10771086.

Fransen E, Van Camp G. The COCH gene: A frequent cause of hearing impairment and vestibular dysfunction? Br J Audiol 1999;33:297-302.

Heining C, Tikka T, Colley S, Zilinskiene L, Coulson C. A Comparison of Cochlear Nerve Size in Normal-Hearing Adults Using Magnetic Resonance Imaging. J Int Adv Otol 2017;13:300-303.

Hildebrand MS, Gandolfo L, Shearer AE, Webster JA, Jensen M, Kimberling WJ, et al. A novel mutation in COCH-implications for genotype-phenotype correlations in DFNA9 hearing loss. Laryngoscope 2010;120:2489-2493.

Hildebrand MS, Tack D, DeLuca A, Hur IA, Van Rybroek JM, McMordie SJ, et al. Mutation in the COCH gene is associated with superior semicircular canal dehiscence. Am J Med Genet Part A 2009;149A:280-285.

Ishiyama G, Lopez IA, Ishiyama P, Vinters HV, Ishiyama A. The blood labyrinthine barrier in the human normal and Meniere's disease macula utricle. Sci Rep. 2017;7(1):253.

Juhn, S. K., Hunter, B. A. \& Odland, R. M. Blood-labyrinth barrier and fluid dynamics of the inner ear. Int Tinnitus Journal 2001;7:72-83.

Kemperman MH, De Leenheer EMR, Huygen PLM, Van Duijnhoven G, Morton CC, Robertson NG, et al. Audiometric, vestibular, and genetic aspects of a DFNA9 family with a G88E COCH mutation. Otol Neurotol 2005;26:926-933. 
Khetarpal U. DFNA9 is a progressive audiovestibular dysfunction with a microfibrillar deposit in the inner ear. Laryngoscope 2000;110:1379-1384.

Kujawa SG, Liberman MC. Adding insult to injury: cochlear nerve degeneration after "temporary" noise-induced hearing loss. J Neurosci 2009;29:14077-14085.

Lemaire FX, Feenstra L, Huygen PLM, Fransen E, Devriendt K, Van Camp G, et al. Progressive late-onset sensorineural hearing loss and vestibular impairment with vertigo (DFNA9/COCH): longitudinal analyses in a belgian family. Otol Neurotol 2003;24:743-748.

Li L, Ikezono T, Watanabe A, Shindo S, Pawankar R, Yagi T. Expression of full-length Cochlin p63s is inner ear specific. Auris Nasus Larynx 2005;32:219-223.

Manolis EN, Yandavi N, Nadol JB, Eavey RD, McKenna M, Rosenbaum S, et al. A gene for non-syndromic autosomal dominant progressive postlingual sensorineural hearing loss maps to chromosome 14q12-13. Hum Mol Genet 1996;5:1047-1050.

Merchant SN, Linthicum FH, Nadol JBJ. Histopathology of the inner ear in DFNA9. Adv Otorhinolaryngol 2000;56:212-217.

Nadol JB, Xu W-Z. Diameter of the cochlear nerve in deaf humans: implications for cochlear implantation. Ann Otol Rhinol Laryngol 1992;101:988-993.

Naganawa S, Yamazaki M, Kawai H, Bokura K, Sone M, Nakashima T. Visualization of endolymphatic hydrops in Ménière's disease after single-dose intravenous gadolinium-based contrast medium: timing of optimal enhancement. Magn Reson Med Sci. 2012;11(1):43-51.

Nagy I, Trexler M, Patthy L. The second von Willebrand type A domain of cochlin has high affinity for type I, type II and type IV collagens. FEBS Lett 2008;582:4003-4007.

Nakashima T, Naganawa S, Pyykkö I, Gibson WPR, Sone M, Nakata S, et al. Grading of endolymphatic hydrops using magnetic resonance imaging. Acta Otolaryngol 2009;129:5-8.

Pakdaman MN, Ishiyama G, Ishiyama A, et al. Blood-Labyrinth Barrier Permeability in Menière Disease and Idiopathic Sudden Sensorineural Hearing Loss: Findings on Delayed Postcontrast 3D-FLAIR MRI. AJNR Am J Neuroradiol. 2016;37(10):1903-1908.

Py BF, Gonzalez SF, Long K, Kim MS, Kim YA, Zhu H, et al. Cochlin produced by follicular dendritic cells promotes antibacterial innate immunity. Immunity 2013;38:1063-1072.

Robertson NG, Cremers CWRJ, Huygen PLM, Ikezono T, Krastins B, Kremer H, et al. Cochlin immunostaining of inner ear pathologic deposits and proteomic analysis in DFNA9 deafness and vestibular dysfunction. Hum Mol Genet 2006;15:1071-1085.

Robertson NG, Lu L, Heller S, Merchant SN, Eavey RD, McKenna M, et al. Mutations in a novel cochlear gene cause DFNA9, a human nonsyndromic deafness with vestibular dysfunction. Nat Genet 1998;20:299-303.

Street VA, Kallman JC, Robertson NG, Kuo SF, Morton CC, Phillips JO. A novel DFNA9 mutation in the vWFA2 domain of $\mathrm{COCH}$ alters a conserved cysteine residue and intrachain disulfide bond formation resulting in progressive hearing loss and site-specific vestibular and central oculomotor dysfunction. Am J Med Genet Part A 2005;139 A:86-95.

Usami S, Takahashi K, Yuge I, Ohtsuka A, Namba A, Abe S, et al. Mutations in the $\mathrm{COCH}$ gene are a frequent cause of autosomal dominant progressive cochleo-vestibular dysfunction, but not of Meniere's disease. Eur J Hum Genet 2003;11:744-748.

Vermeire K, Brokx JPL, Wuyts FL, Cochet E, Hofkens A, De Bodt M, et al. Good speech recognition and quality-of-life scores after cochlear implantation in patients with DFNA9. Otol Neurotol 2006;27:44-49. 


\section{Figure Legends}

Fig. 1. MR findings in two subjects affected by DFNA9 $(A, B, C, D)$ and SSHL $(E, F, G, H)$. $(A, E)$ axial precontrast T1 weighted fast spin echo (T1 FSE), (B,F) T2 weighted three-dimensional (3D) steadystate free precession (FFSP) sequence, $(C, G)$ three-dimensional (3D) Fluid-Attenuated Inversion Recovery (FLAIR) sequence and (D,H) 4hs-delayed postcontrast 3D-FLAIR sequence.

On T1 FSE and 3D-FFSP no signal abnormalities are present in both DFNA9 patient $(A, B)$ and SSHL patient $(E, F)$. On pre-contrast 3D-FLAIR sequence, SSHL patient shows mild signal hyperintensity on the right affected ear (arrow) indicating proteinaceous content in the perilymph (G), in contrast DFNA9 patient shows no signal abnormalities (C). On 4hs-delayed 3D-FLAIR sequence, the SSHL patient shows mild enhancement in the affected right ear (dotted arrow) indicating the presence of Blood-Labyrinthine Barrier (BLB) breakdown, and no signal abnormalities are present in the left healthy ear $(\mathrm{H})$; in contrast the DFNA9 patient shows prominent bilateral enhancement of the inner ear (arrow) suggesting bilateral BLB breakdown (D). The metrics calculated in the SSHL patient are the follows: T1 cSI -> R 0.6 - L 0.7; T2 cSI -> R 0.7 - L 0.7 ; FLAIR cSI -> R 4.6 - L 3.4; Enhancement -> R $3.3-\mathrm{L} 1.5$. The metrics calculated in the DFNA9 patient are listed in Table 4, patient \#4.

Fig. 2. (A) axial T2 weighted three-dimensional (3D) steady-state free precession (FFSP) image taken at the level of the cochlea showing position of the hand-drawn region of interest (ROI) contouring the middle turn of the cochlea. A second ROI of the same area has been drawn in the cerebellopontine angle (CPA) to depict liquor signal intensity (S.I.), used as reference value for calculation of signal intensity ratio as described in "Material and Methods" section. (B) axial 4hsdelayed postcontrast three-dimensional (3D) Fluid-Attenuated Inversion Recovery (FLAIR) image taken at the level of the cochlear basal turn and vestibular labyrinth. Image shows the hand-drawn region of interest (ROI) contouring the vestibular endolymphatic space (arrow) and the hand-drawn $\mathrm{ROI}$ contouring the sum of vestibular perilymphatic and endolymphatic space (arrow head) used for the quantitative assessment of endolymphatic hydrops as described by Nakashima et al., [2009] [Nakashima et al., 2009]. 